ENCYCLOPÉDIE Encyclopédie berbère

BERBERE

5 | 1988

5 | Anacutas - Anti-Atlas

\title{
Anthropologie (Partie I)
}

\author{
M.-C. Chamla et D. Ferembach
}

\section{OpenEdition}

Journals

Édition électronique

URL : http://journals.openedition.org/encyclopedieberbere/2523

DOI : 10.4000/encyclopedieberbere.2523

ISSN : 2262-7197

\section{Éditeur}

Peeters Publishers

\section{Édition imprimée}

Date de publication : 1 avril 1988

Pagination : 713-775

ISBN : 2-85744-379-6

ISSN : 1015-7344

\section{Référence électronique}

M.-C. Chamla et D. Ferembach, « Anthropologie (Partie I) », Encyclopédie berbère [En ligne], 5 | 1988, document A234, mis en ligne le 01 décembre 2012, consulté le 14 octobre 2020. URL : http://

journals.openedition.org/encyclopedieberbere/2523; DOI : https://doi.org/10.4000/ encyclopedieberbere. 2523

Ce document a été généré automatiquement le 14 octobre 2020

(c) Tous droits réservés 


\title{
Anthropologie (Partie I)
}

\author{
M.-C. Chamla et D. Ferembach
}

1 L'origine anthropologique des Berbères doit être recherchée dans les temps préhistoriques. Ce n'est qu'à partir de l'Épipaléolithique qu'apparaissent des types humains qu'il est raisonnable de compter parmi les ancêtres des populations actuelles du Maghreb et du Sahara.

\section{Époque épipaléolithique (M.-C. Chamla)}

2 La complexité de l'Épipaléolithique du Maghreb a été maintes fois soulignée par les préhistoriens. Actuellement, on distingue deux grands ensembles culturels, l'Ibéromaurusien et le Capsien qui ne sont pas exactement contemporains et présentent différentes phases d'évolution. D'autres cultures épipaléolithiques distinctes de ces deux ensembles (Columnatien, Mellalien, Kérémien...) ont une extension moindre.

3 La civilisation ibéromaurusienne représentée dans des gisements généralement situés dans les zones littorale et tellienne s'étend sur plusieurs millénaires. Les dates les plus anciennes actuellement connues vont de 13750 B.C. à Taforalt à environ 7590 B.C. à El Hamel (Camps, 1973 et 1974). La chronologie capsienne dont on possède de nombreuses datations a été estimée de façon précise assez récemment. Les dates les plus anciennes sont celles des gisements d'Aïn Naga (7 350 B.C.) et de Medjez II (6910 B.C.). Les dates les plus récentes ne dépassent pas 4500 ans B.C. environ (El Mermouta). Le terme d'Épipaléolithique englobe donc une période très longue allant du XIV millénaire au ve millénaire.

4 Les ossements humains exhumés des gisements ibéromaurusiens et capsiens ont fait l'objet de nombreuses descriptions (cf. liste de ces gisements au tableau I). Rappelons que l'Afrique du Nord était habitée durant l'Épipaléolithique par une population à caractéristiques robustes comme l'étaient vers la même période les habitants de certaines régions de l'Europe et du Proche-Orient. On y rencontrait deux types fondamentalement différents, l'un de morphologie classiquement décrite comme cromagnoïde, dénommé type de Mechta-Afalou, présent dans les gisements ibéromaurusiens et certaines sépultures capsiennes, l'autre très différent et arrivé vers 
le $\mathrm{VII}^{\mathrm{e}}$ millénaire, à affinités méditerranéennes, des Protoméditerranéens dont on trouve les premières traces dans les gisements capsiens.

\section{Les hommes des nécropoles ibéromaurusiennes}

5 H.V. Vallois a défini, le premier, la morphologie des hommes du type de Mechta-Afalou, d'après l'étude d'une cinquantaine d'individus recueillis dans l'abri-sous-roche d'Afalou-bou-Rhummel* (in Arambourg, etc., 1934) et dont la morphologie est bien connue et très caractéristique. Les traits principaux en sont les suivants : robustesse générale, forte épaisseur des parois crâniennes, grandes dimensions du crâne et de l'ensemble du squelette, tendance à la mésocéphalie, face large et courte munie d'arcades sus-orbitaires saillantes réunies en un bourrelet médian, orbites basses et rectangulaires, mandibule vaste, au corps très divergent avec projection latérale des gonions, menton accusé, denture assez volumineuse et atteinte de lésions pathologiques nombreuses. La stature de ces hommes était élevée (1,77 m en moyenne), leurs épaules larges, leur squelette très robuste. La comparaison des hommes et des femmes de ces gisements montre qu'il existait un dimorphisme sexuel prononcé, particularité fréquente chez les populations préhistoriques et notamment au Mésolithique. En outre, ils pratiquaient une mutilation dentaire, l'ablation des incisives médianes supérieures.

Cette description donne une image stéréotypée des hommes ibéromausuriens d'Afrique du Nord. En fait, la comparaison des hommes trouvés dans différents gisements indique que l'unicité du type de Mechta-Afalou était loin d'être parfaite.

Crânes d'époque ibéromaurusienne : 1) Crâne féminin $\mathrm{H} 13$ d'Afalou bou Rhumel ; 2) Crâne masculin de Champlain ; 3) Crâne masculin H 1 de Gambetta.

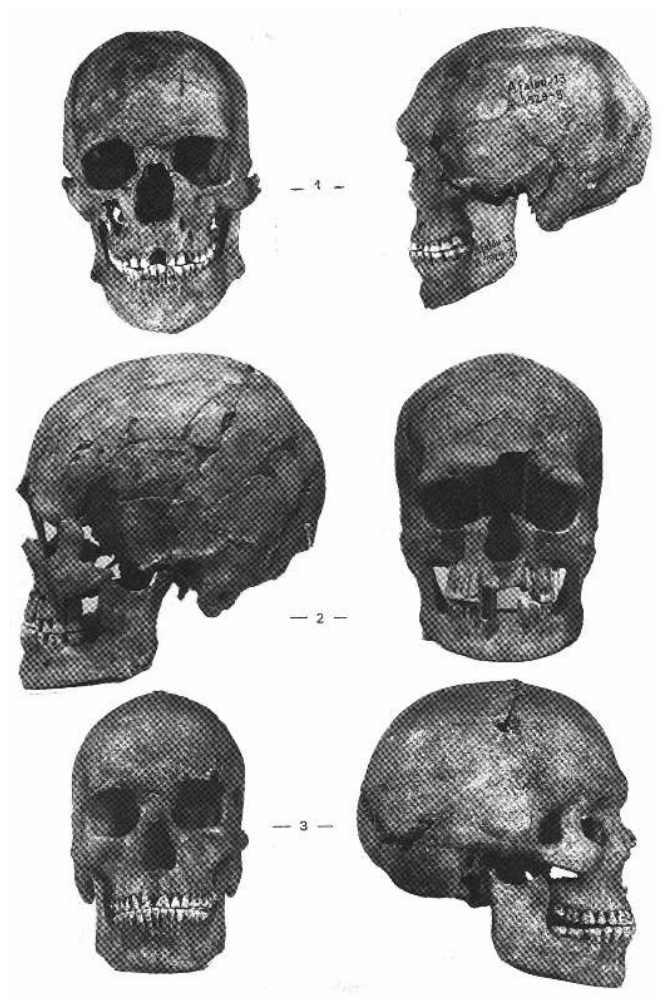


7 Déjà les restes trouvés à Afalou montrent des différences morphologiques, notamment une tendance chez quelques sujets à une mésocéphalie prononcée ou une brachycéphalie accompagnée d'une certaine atténuation des reliefs osseux avec moindre proéminence des diverses saillies osseuses et du bourrelet sus-orbitaire. En outre, on y trouve quelques individus à face longue et orbites basses. Des restes recueillis dans d'autres gisements montrent également quelques variations. Notamment les hommes de Taforalt* (Maroc) décrits par D. Ferembach (1962) forment un groupe sensiblement plus homogène, avec une robustesse encore plus marquée que chez les hommes d'Afalou. Ceux, plus récents, datant d'une époque contemporaine du Capsien (Colummatien, 6000 à 5000 B.C.), trouvés dans la nécropole de Columnata dans l'Ouest algérien (Chamla, 1970), montrent des signes de gracilisation et de brachycéphalisation par rapport aux hommes plus anciens d'Afalou et de Taforalt, et sont qualifiés du terme de "mechtoïdes». Ils présentent une moindre robustesse générale, des dimensions du crâne et des os longs moins grandes (stature, 1,72 m en moyenne chez les hommes), une tendance à la méso-brachycéphalie, des reliefs osseux moins développés, une denture moins volumineuse, toutes caractéristiques qui dénotent une gracilisation par rapport aux restes ibéromausuriens plus anciens. L'usure des dents était chez eux moins précoce et moins intense, la carie était en augmentation notable, indiquant des modifications probables dans le régime alimentaire et une moindre résistance aux facteurs cariogènes que leurs prédécesseurs. Enfin, on constate chez eux l'extension aux deux maxillaires et aux huit incisives de l'avulsion dentaire qui pourrait indiquer des contacts avec leurs contemporains trouvés dans les gisements capsiens d'Algérie chez qui cette pratique était courante. D'autres restes, peu nombreux, recueillis dans quelques gisements ibéromausuriens, dont certains sont d'époque peut-être capsienne, présentent une morphologie analogue. Les uns (Kef Oum Touiza, Djebel Taya, Champlain) sont comparables aux hommes d'Afalou, d'autres (La Mouillah*, Ali-Bacha*) se rapprocheraient des hommes de Columnata*. Par ailleurs, l'escargotière de Gambetta a livré les restes complets de deux individus, un homme de type protoméditerranéen robuste et une femme mechtoïde. Enfin, dans un gisement ibéromausurien non douteux, Rachgoun, a été trouvée, cas unique jusqu'ici, parmi des sujets de morphologie mechtoïde, une femme de type méditerranéen gracile.

8 Les caractères physiques des hommes de Mechta-Afalou ont été maintes fois rapprochés de ceux des Cro-magniens européens avec qui certains auteurs pensent qu'ils auraient eu des contacts, voire même une origine commune. Cette hypothèse semble devoir être abandonnée. Outre le fait que les hommes d'Afalou et de Tafolralt diffèrent par toute une série de caractères des hommes du début et de la fin du Paléolithique supérieur européen - notamment une largeur du crâne et des dimensions frontales plus élevées, une hauteur de la voûte nettement plus forte, une largeur faciale plus grande, des orbites moins basses, un nez plus large, enfin des indices crâniens et faciaux différents et une stature plus grande - la découverte récente dans des niveaux atériens, plus anciens que les couches ibéromausuriennes (environ 20000 ans B.C.) au Maroc à Dar-es-Soltane 2 (A. Debenath, 1976) et à Temara (J. Roche, 1976), montre que la présence, sur le sol africain, d'hommes du type de Mechta-Afalou est beaucoup plus ancienne qu'on ne l'avait pensé. Notamment l'homme $\mathrm{n}^{\circ} 5$ de Dar-es-Soltane s'apparente, semble-t-il, à une forme très robuste du type Mechta-Afalou, indiquant que les hommes des gisements ibéromausuriens ne correspondent pas à des éléments nouvellement arrivés à l'époque épipaléolithique, mais qu'ils pourraient résulter d'une évolution sur place d'un type humain plus ancien. 
Les hommes moustériens marocains du Djebel Irhoud présentent en effet une association de caractères des hommes d'Afalou et de traits néandertaliens (Ferembach, op. cit.).

Crânes d'époque capsienne : 1) Crâne masculin de l'Aïn Dokkara ; 2) Crâne masculin H X de Mechta el-Arbi (Fleurance) ; 3) Crâne féminin F 3 de Mechta el-Arbi (série Cole).

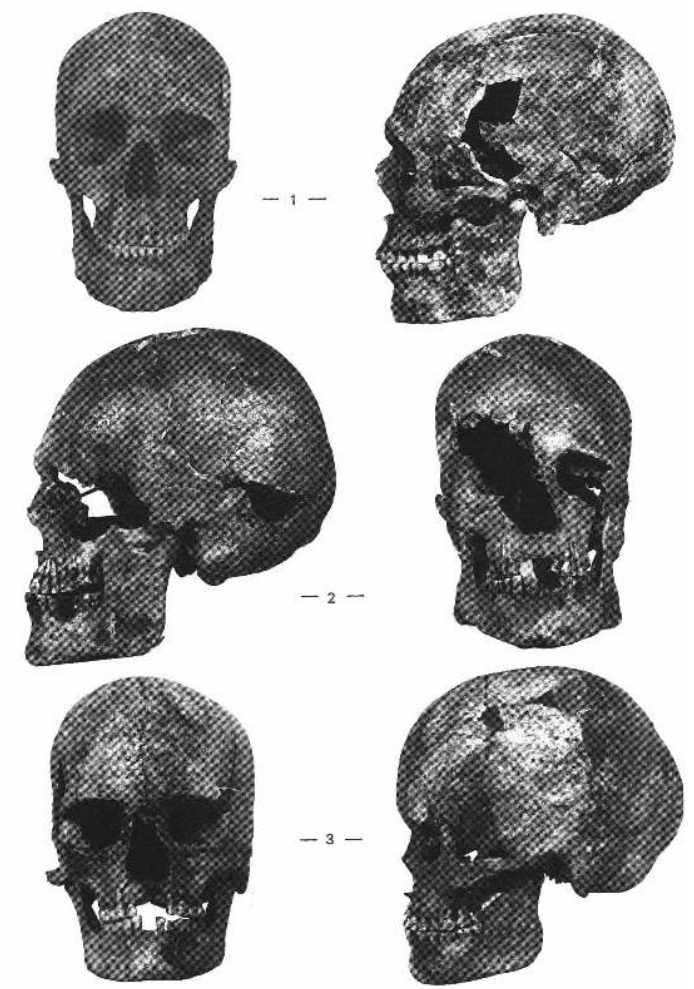

\section{Les hommes des escargotières capsiennes}

Concentrés surtout dans la région orientale de l'Algérie, les gisements capsiens qui présentent généralement l'aspect d'escargotières* cendreuses ont livré de nombreux squelettes dont la morphologie offre une grande diversité indiquant que la population de cette époque était indiscutablement composite (Chamla, 1975). On y trouve deux types fondamentalement différents, l'un dont la morphologie est du type de MechtaAfalou, l'autre d'affinités méditerranéennes, et deux variantes au moins du second élément protoméditerranéen. Ce dernier a été décrit par Vallois (Aïn Méterchem, 1950), Briggs (1955), Chamla (Aïn Dokkara, 1973) et ses deux variantes ont été précisées dans ce dernier travail : l'une (type 1) comprenant des sujets dolicho- à mésocrânes, à face longue et à voûte élevée, aux orbites méso- à hypsiconques, au nez méso- à leptorhinien, orthognathes ou modérément prognathes. L'autre variante (type 2) groupant des sujets dolichocrânes, à voûte basse, à la face de hauteur moyenne, aux orbites mésoconques, au nez mésorhinien, éventuellement prognathes. Chez les deux types la stature était élevée chez les hommes (moyenne 1,76 m), sensiblement plus petite chez les femmes (moyenne 1,63 m) qui présentaient en outre une certaine gracilité comparativement aux hommes nettement plus robustes. Parmi les restes dont on possède un signalement suffisamment complet pour évaluer leurs affinités morphologiques, on constate que, contrairement à une opinion assez répandue, le type 
de Mechta-Afalou est loin d'être rare dans les gisements capsiens, mais que, sauf à Medjez II où il semble qu'on puisse considérer comme mechtoïde la femme $\mathrm{n}^{\circ} 4$ et les trois autres sujets comme des Protoméditerranéens, il existe une séparation assez tranchée des deux éléments qui n'apparaissent pas coexister dans les gisements capsiens découverts à ce jour. Dans le tableau II est indiquée une répartition des différents types dans ces gisements. Sur un total de 24 individus, la proportion de sujets appartenant au type de Mechta-Afalou est de 10, soit $42 \%$, pour 14 du type protoméditerranéen, soit $58 \%$. Bien que le nombre de sujets protoméditerranéens soit encore insuffisant pour que l'on puisse tirer des conclusions générales, il semble que la première variante à face longue et voûte élevée soit davantage représentée que la seconde à face moyenne.

10 Chez les hommes comme chez les Ibéromaurusiens, la mutilation dentaire était une pratique courante. L'avulsion mixte des incisives au maxillaire et à la mandibule était généralement pratiquée, plus souvent chez les femmes que chez les hommes (pourcentage d'avulsions chez 15 hommes, 46,6\%, chez 18 femmes 77,7 \%).

11 Les hommes protoméditerranéens des gisements capsiens d'Afrique du Nord ne sont pas comparables aux hommes mésolithiques d'Europe méridionale, notamment ceux de Muge et de la région pyrénéenne de Montardit caractérisés par un squelette nettement moins robuste, une face de hauteur moyenne et des orbites plutôt basses. Ils se rapprocheraient des hommes protoméditerranéens robustes du Natoufien terminal de Palestine.

\section{Époque néolithique}


Crânes d'époque néolithique : 1) Crâne masculin d'Aïn Meterchem (Néolithique ou Capsien supérieur) ; 2) Crâne masculin de l'Oued Guettara ; 3) Crâne masculin de Tafaraoui.

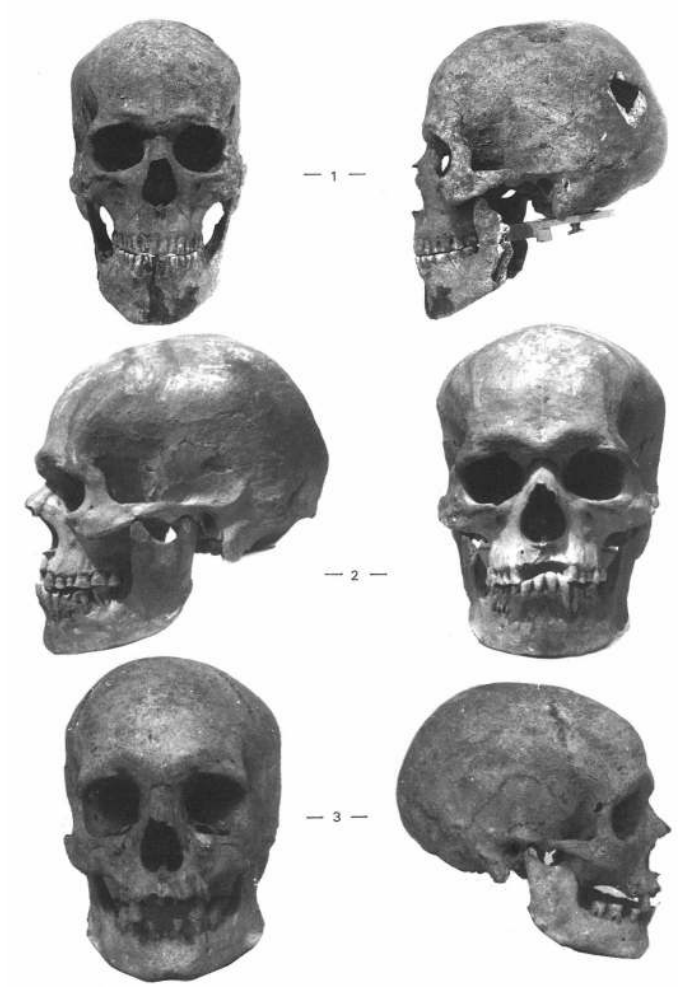

Ce type protoméditerranéen robuste ne prédominera pas très longtemps en Afrique du Nord. On le retrouve au Néolithique ( $v^{\mathrm{e}}-\mathrm{III} \mathrm{e}^{\mathrm{e}}$ millénaire), parallèlement à des hommes plus graciles de type méditerranéen différent du premier et à des éléments du type de Mechta-Afalou, surtout localisés à l'ouest du pays. En outre, les premiers individus de type négroïde font leur apparition dans l'Est. Dans le tableau III sont indiquées les affinités des individus dont on possède un signalement suffisant. Les différences entre les régions occidentale et orientale sont très nettes. Dans l'ouest les individus du type Mechta-Afalou prédominent avec, chez certains, une robustesse particulièrement accentuée, notamment à Brédéah (Oued Guettura), aux Troglodytes, Rio Salado, Mougharet el Aliya, Gar Cahal. Leur stature semble un peu moins élevée que celle des hommes des époques précédentes. Des hommes de type méditerranéen robuste, peu nombreux, sont également représentés. En revanche, à l'est, si quelques hommes du type de Mechta-Afalou subsistent encore, le type protoméditerranéen est prédominant, les uns robustes et analogues aux hommes des gisements capsiens, quoique moins grands (moyenne, 1,71 m), les autres graciles. Ces derniers constituent un élément différent du type robuste. Particulièrement graciles, ils sont de petite taille $(1,55 \mathrm{~m}$ à 1,69 m chez les hommes), leur dimorphisme sexuel est faible, et ils présentent souvent un chignon occipital, un prognathisme alvéolaire et une légère platyrhinie associés à une face étroite, une dépression sus-nasale peu marquée. Nouveaux venus, semble-t-il, on ignore leur origine. Le fait qu'une série d'individus présentant cette morphologie ait été trouvée dans des gisements néolithiques des grottes de l'Aurès (Khenchela, Batna) ne signifie pas qu'ils représentaient un élément prédominant sur le type protoméditerranéen robuste dans la région orientale. Il faut attendre d'autres découvertes avant de se prononcer. Nouveaux venus également sont les quelques individus de type négroïde découverts dans le gisement de Redeyef en Tunisie et dans 
la grotte de Khenchela conjointement avec des Méditerranéens. Chez ces hommes néolithiques, la pratique de l'avulsion dentaire a quasiment disparu à l'est (1 homme sur 28 sujets), alors qu'elle s'est maintenue à l'ouest où, d'après des observations personnelles, on trouve des traces de mutilation chez 71 \% d'un total de 42 individus, avec une proportion sensiblement égale d'hommes et de femmes présentant deux types d'avulsion, mixte ou localisée au maxillaire seulement.

Localisation des gisements préhistoriques cités dans le texte.

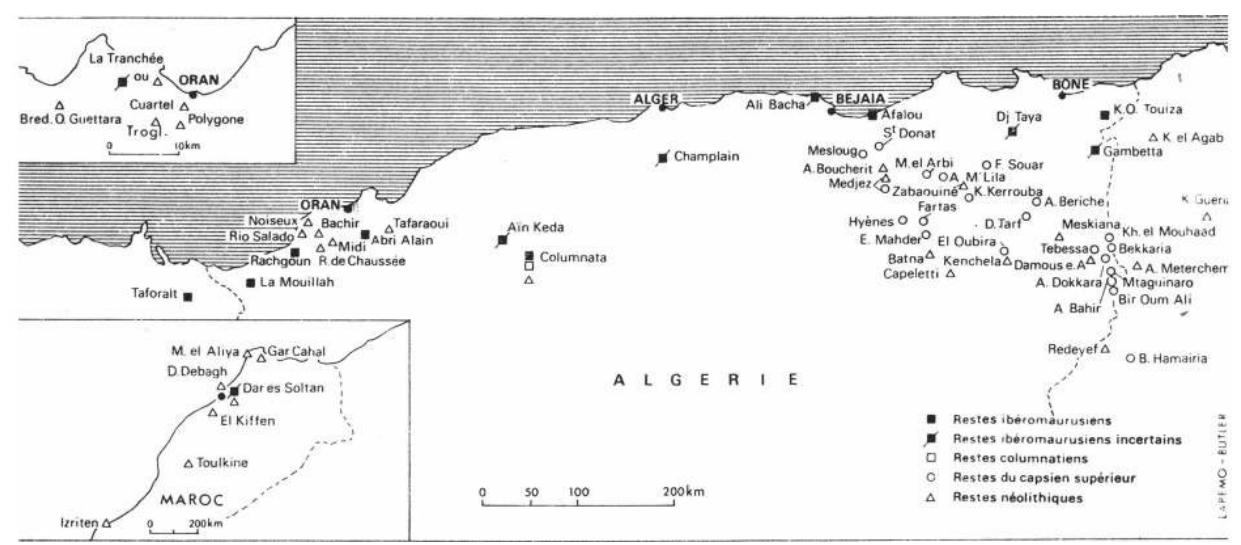

\section{BIBLIOGRAPHIE}

\section{Epoque épipaleolithique}

ARAMBOURG C. BOULE M., VALLOIS H. et VERNEAU R., Les grottes paléolithiques de Beni Seghoual (Algérie), Archives de l'Inst. paléont. hum., mémoire n 13, Paris, 1934, 242 p.

BALOUT L., « Les hommes préhistoriques du Maghreb et du Sahara. Inventaire descriptif et critique ", Libyca, 1954, t. 2, 461 p.

BALOUT L. et BRIGGS L.C., « Débris humains de l'escargotière de Gambetta », Bull. soc. d'hist. nat. de l'Afrique du Nord, 1949, t. 40, p. 127.

BALOUT L. et BRIGGS L.C., " Tête osseuse du Kef oum Touiza », Bull, d'hist. nat. d'Afrique du Nord, 1949, t. 40 , pp. $64-70$.

BALOUT L. et BRIGGS L.C., « Mechta el Arbi », Trav. labor, d'anthrop. et d'arch. préh., musée de Bardo, Alger, 19512, n III-IV, $131 \mathrm{p}$.

BERTHOLON, « Notes sur 4 crânes humains trouvés par M. Debruge à Tébessa », VII congrès préhist. de France, Nîmes, 1911, p. 214.

BRIGGS L.C., « Tête osseuse du Khanguet el Mouhaad (fouilles J. Morel) », Libyca, 1953, t. 1, pp. 120-140.

BRIGGS L.C., « Deux têtes osseuses de la collection Debruge, le crâne "type" de Mechta el Arbi et le crâne "A" de la grotte des Hyènes ", Libyca, 1954, t. 2, pp. 121-149. 
BRIGGS L.C., « The stone age races of Northwest Africa », American school of prehistoric research, Peabody Mus., bull. 18, 1955, 98 p.

CAMPS G., Les civilisations préhistoriques de l'Afrique du Nord et du Sahara, Paris, Doin, 1974, 366 p. CAMPS G., DELIBRIAS G. et THOMMERET J., « Chronologie des civilisations préhistoriques du nord de l'Afrique d'après le radiocarbone ", Libyca, 1973, t. 21, pp. 65-89.

CHAMLA M.-C, « Note sur les restes humains (H4) découverts à Rachgoun en février 1964 » in Camps G., « Le gisement de Rachgoun, Oranie », Libyca, 1966, t. XIV, pp. 182-188.

CHAMLA M.-C, « Les hommes épipaléolithiques de Columnata (Algérie occidentale). Étude anthropologique », Mém. centre rech. anthrop. préhist., ethonogr., Alger, 1970, n XV, 132 p.

CHAMLA M.-c, «Étude anthropologique de l'Homme capsien de l'Aïn Dokkara (Algérie orientale) », Libyca, 1973, t. XXI, pp. 9-53.

CHAMLA M.-C, «Les Algériens et les populations arabo-berbères du nord de l'Afrique », Mém. du C.R.A.P.E., Alger, 1974, $\mathrm{n}^{\circ} \mathrm{XXIV}, 128 \mathrm{p}$.

CHAMLA M.-C, « La diversité des types humains dans les gisements capsiens », in Camps-Fabrer H., Un gisement capsien de faciès sétifien, Medjez II, EL Eulma, Algérie, édit. C.N.R.S., Paris, 1975, pp. 373-376.

CHAMLA M.-C, « Les hommes des sépultures protohistoriques et puniques d'Afrique du Nord (Algérie et Tunisie) ", L'anthropologie, 1975, n 4, pp. 659-692, et 1976, nº 1, pp. 75-116.

CHAMLA M.-C, « Les hommes cromagnoïdes d'Afrique du Nord et le problème de leur extinction », IXe congrès intern, des Sc. préh. etprotoh., Nice, 1976, coll. II, pp. 144-152 (prétirage).

CHARON M., ORTLIEB L. et PETIT-MAIRE N., « Occupation humaine holocène de la région du cap Juby (sud-ouest marocain), (Izriten) », Bull, et mém. soc. d'anthrop. de Paris, 1973, n 4, pp. 379-412.

DELISLE F., " Note sur les ossements humains de la grotte "Ali Bacha" ", Assoc. fr. pour l'avancement des sciences, Montauban, 1902, t. 2, pp. 883-885.

DEMOULIN F., Le crâne des Algériens, thèse fac. sciences, Paris, 1972, 84 p.

ENNOUCHI E., « Découverte d'un homme de Mechta à Rabat (Douar Debagh) », L'Anthropologie, 1953, pp. 272-283.

FEREMBACH D., La nécropole épipaléolithique de Taforalt (Maroc oriental), Paris, 1962, 171 p.

FEREMBACH D., «Les restes humains de Témara », Bull, et mém. soc. d'anthrop. de Paris, 1976, nº 2 , pp. $175-180$.

FEREMBACH D., «Les restes humains de la grotte de Dar-es-Soltane 2 (Maroc) », Bull, et mém. soc. d'anthrop. de Paris, n 2, pp. 183-193.

FUSTÉ M., « Restos humanos procedentes de la Cueva de Car Cahal (Marruecos) », Trabajos Inst. Bernard, de Sahagun, 1961, n 3, pp. 155-184.

LEBLANC E., «L'Homme du djebel Fartas », Rec. not. et mém. socv. archéol. de Constantine, 1922-1923, t. 54, pp. 143-152.

MARCHAND H., « Les hommes fossiles de la Mouillah (Oran) », Rev. anthrop., 1936, t. 46, pp. 239-253. MARCHAND H., « Les documents humains de l'escargotière d'Ain Bahir », Bull. mém. soc. archéol. de Constantine, 1934, $20 \mathrm{p}$. 
MAYET L., « Crâne masculin de la grotte du Djebel Taya », Bull. mens. soc. Linn., 1944, nº 7-8, pp. 108-110.

PENROSE L. S., « Distance, size and shape », Annals of Eugenics, 1954, t. 18, p. 337-343.

VALloIS H., « Le squelette d'Aïn Méterchem », Atti del I Congr. de Preist. e Protoistor. médit., Florence, 1950, pp. 103-104.

VALLOIS H., « Les restes humains de Dar-es-Soltan » in Ruhlmann, La grotte préhistorique de Dar-esSoltan, 1951, pp. 179-202.

VALLOIS H., « Note sur les ossements humains de la grotte du Kef el Agab (Tunisie) », Libyca, 1953, t. L, pp. 271-308.

VALLOIS H., « Le crâne-trophée capsien de Faïd Souar II, Algérie », L'Anthropologie, 1971, pp. 191-220, et pp. 397-414.

INDEX

Mots-clés : Préhistoire, Anthropologie, Sahara, Protohistoire, Maghreb, Origines, Histoire contemporaine 